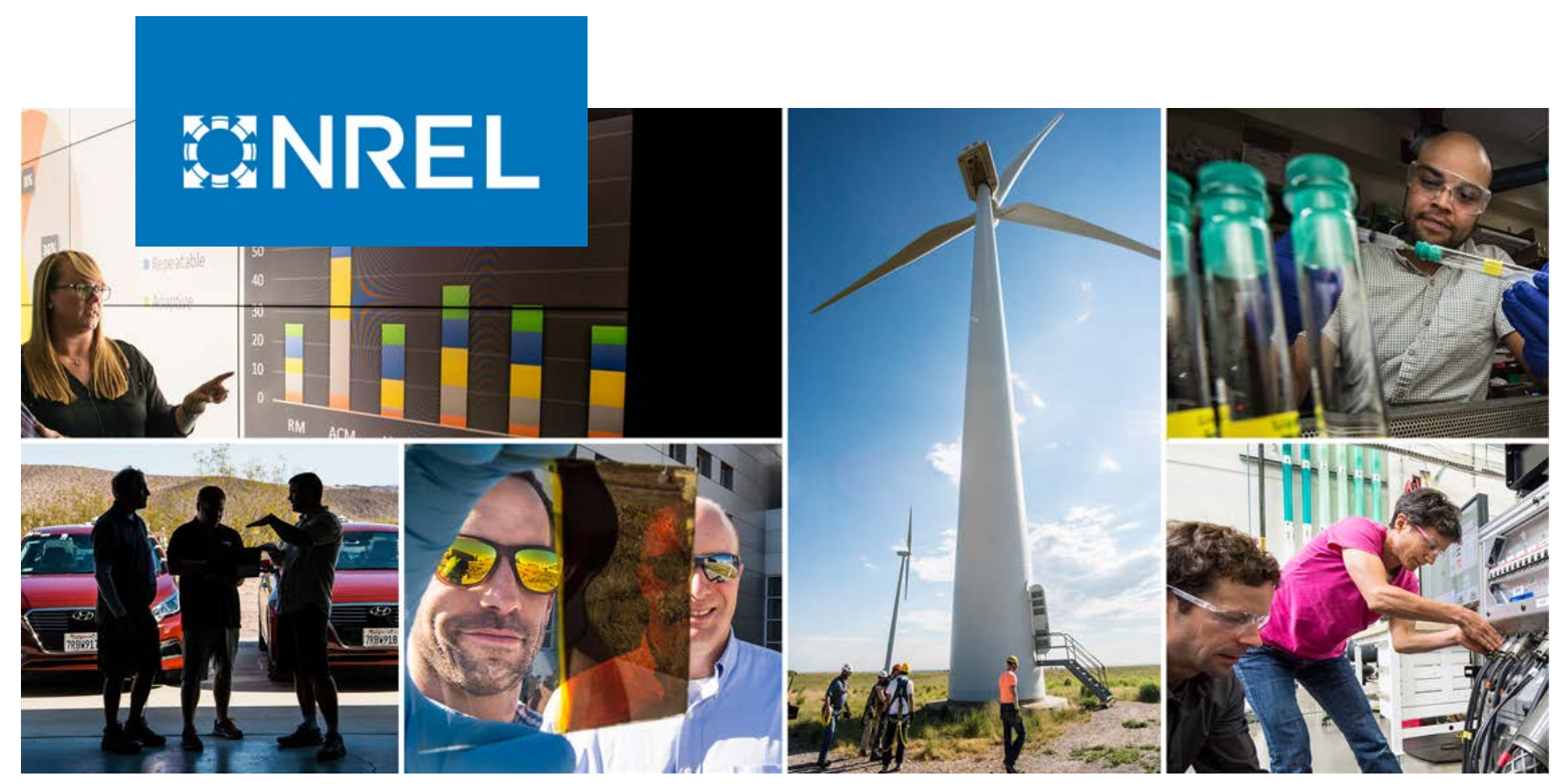

\title{
NREL's HydroGEN Photoreactor Platform Design and Characterization
}

Chase W. Aldridge, Ellis W. Klein, William A. Callahan, Keenan Wyatt, Todd G. Deutsch, and James L. Young

National Renewable Energy Laboratory

NREL is a national laboratory of the U.S. Department of Energy

Office of Energy Efficiency \& Renewable Energy

Operated by the Alliance for Sustainable Energy, LLC

This report is available at no cost from the National Renewable Energy Laboratory (NREL) at www.nrel.gov/publications.
Technical Report

NREL/TP-5900-79644

September 2021 


\title{
GNREL
}

\section{NREL's HydroGEN Photoreactor Platform Design and Characterization}

\author{
Chase W. Aldridge, Ellis W. Klein, William A. Callahan, \\ Keenan Wyatt, Todd G. Deutsch, and James L. Young \\ National Renewable Energy Laboratory
}

\section{Suggested Citation}

Aldridge, Chase W., Ellis W. Klein, William A. Callahan, Keenan Wyatt, Todd G. Deutsch, and James L. Young. 2021. NREL's HydroGEN Photoreactor Platform Design and Characterization. Golden, CO: National Renewable Energy Laboratory. NREL/TP-590079644. https://www.nrel.gov/docs/fy21osti/79644.pdf.

NREL is a national laboratory of the U.S. Department of Energy Office of Energy Efficiency \& Renewable Energy Operated by the Alliance for Sustainable Energy, LLC

This report is available at no cost from the National Renewable Energy Laboratory (NREL) at www.nrel.gov/publications.

Contract No. DE-AC36-08G028308
Technical Report NREL/TP-5900-79644

September 2021

National Renewable Energy Laboratory 15013 Denver West Parkway Golden, CO 80401 303-275-3000 • www.nrel.gov 


\section{NOTICE}

This work was authored by the National Renewable Energy Laboratory, operated by Alliance for Sustainable Energy, LLC, for the U.S. Department of Energy (DOE) under Contract No. DE-AC36-08GO28308. Funding provided by the U.S. Department of Energy Office of Energy Efficiency and Renewable Energy Hydrogen and Fuel Cell Technologies Office. The views expressed herein do not necessarily represent the views of the DOE or the U.S. Government.

This report is available at no cost from the National Renewable Energy Laboratory (NREL) at www.nrel.gov/publications.

U.S. Department of Energy (DOE) reports produced after 1991 and a growing number of pre-1991 documents are available free via www.OSTI.gov.

Cover Photos by Dennis Schroeder: (clockwise, left to right) NREL 51934, NREL 45897, NREL 42160, NREL 45891, NREL 48097, NREL 46526.

NREL prints on paper that contains recycled content. 


\section{Abstract}

This technical report details the design, fabrication, and construction of a flow-through photoreactor developed at the National Renewable Energy Laboratory (NREL). The photoreactor is designed to facilitate indoor and outdoor testing on photoelectrochemical and integrated solar water-splitting devices.

The photoreactor design described here is based on a previous demonstration ${ }^{1}$. The photoreactor version described herein contains several advanced design features that include:

1) Chassis-chuck two-part design

- Quick interchange of photoelectrode samples

- Flexible sample mounting options allowed by the sample mounting chuck

2) Separable counter electrode compartments (here, the counter electrodes are anodes)

3) Reduced electrode separation to lower the electrolyte resistance

4) Improved flow pattern for bubble removal

5) Mounting for spectroradiometer receptor

6) Fresnel lens and collimating tube attachments

The photoreactor presented here is designed primarily to accommodate photocathode materials in an illuminated compartment with a dark anode as the oxygen evolution catalyst. Materials were therefore chosen to be compatible with an acidic environment. Other embodiments could accommodate a photoanode with dark hydrogen evolution catalyst and other build materials for alkaline compatibility.

\section{A video demonstrating photoreactor assembly is available at: https://youtu.be/zmYkbJJAkkI}




\section{Table of Contents}

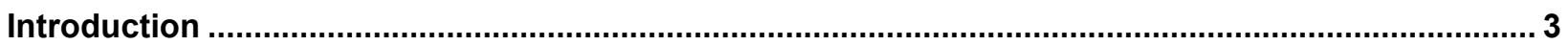

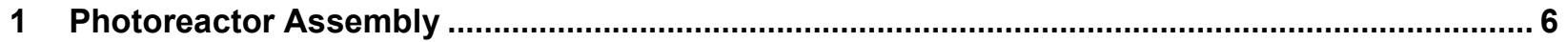

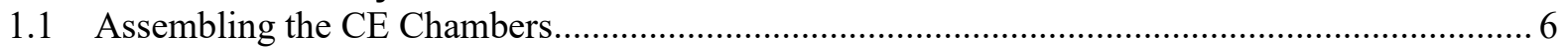

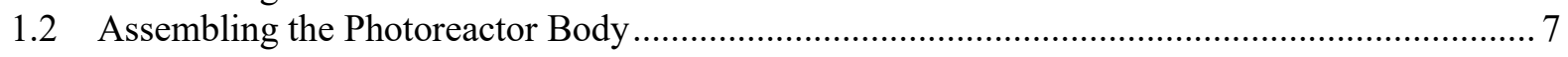

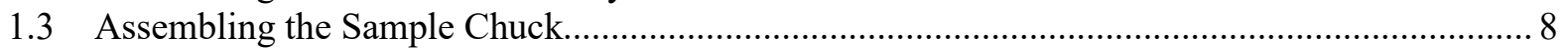

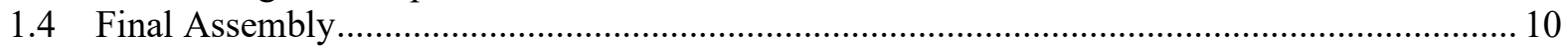

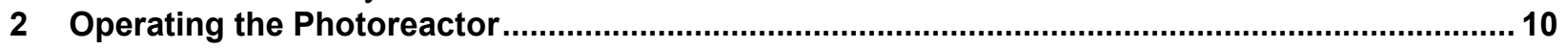

2.1 Measurement Preparation and Chuck Exchange .............................................................. 10

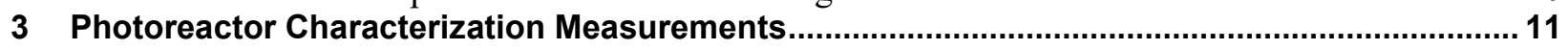

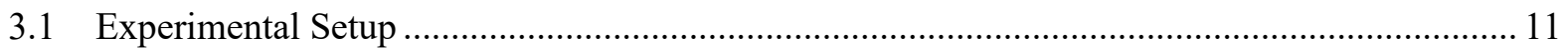

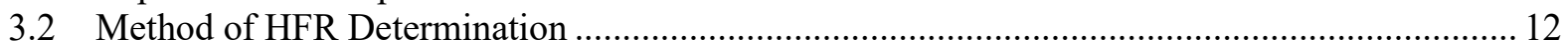

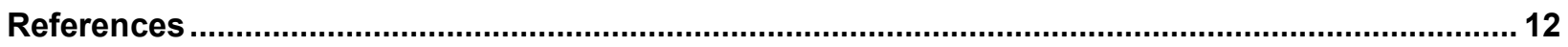

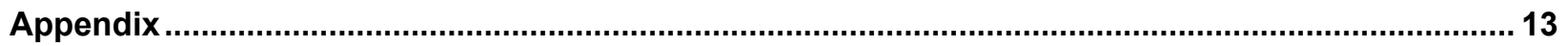

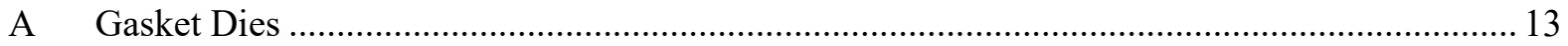

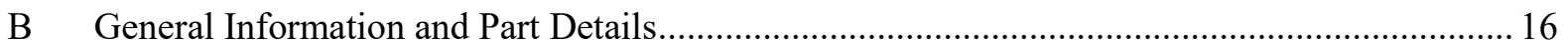




\section{Introduction}

The study of photoelectrochemical devices has for a long time been confined to laboratory benchtops and indoor simulated light sources, but progressions in the field have dictated that a robust outdoor testing apparatus be designed. The chassis-chuck design presented here allows for customizable sample mounting chucks to be employed and for premounted samples to easily be shipped between collaborators. Another benefit of the removable sample chuck is that it greatly reduces the down time between measurements, increasing the throughput of samples and allowing greater utilization of the time periods with good solar and weather conditions.

For on-sun benchmarking measurements, attachments can be mounted to the chassis independently of the sample chuck. Such attachment examples include a collimating tube, Fresnel lens, digital microscope, and/or dark shrouding. To mitigate the accumulation of bubbles between the window and the photoelectrode (PE), the PE chamber has been designed for a high flow rate of electrolyte over the PE surface and elicits a pressure drop between the single inlet line and the and two outlet lines. The last noteworthy focus of this design is that the distance between the PE and each counter electrode (CE) is approximately $5-15 \mathrm{~mm}$, depending on PE geometry and placement, to reduce losses from electrolyte resistance. For the rest of the report, we will refer to the center chamber as the PE chamber and those adjacent as the left and right CE chambers (Figure 1).

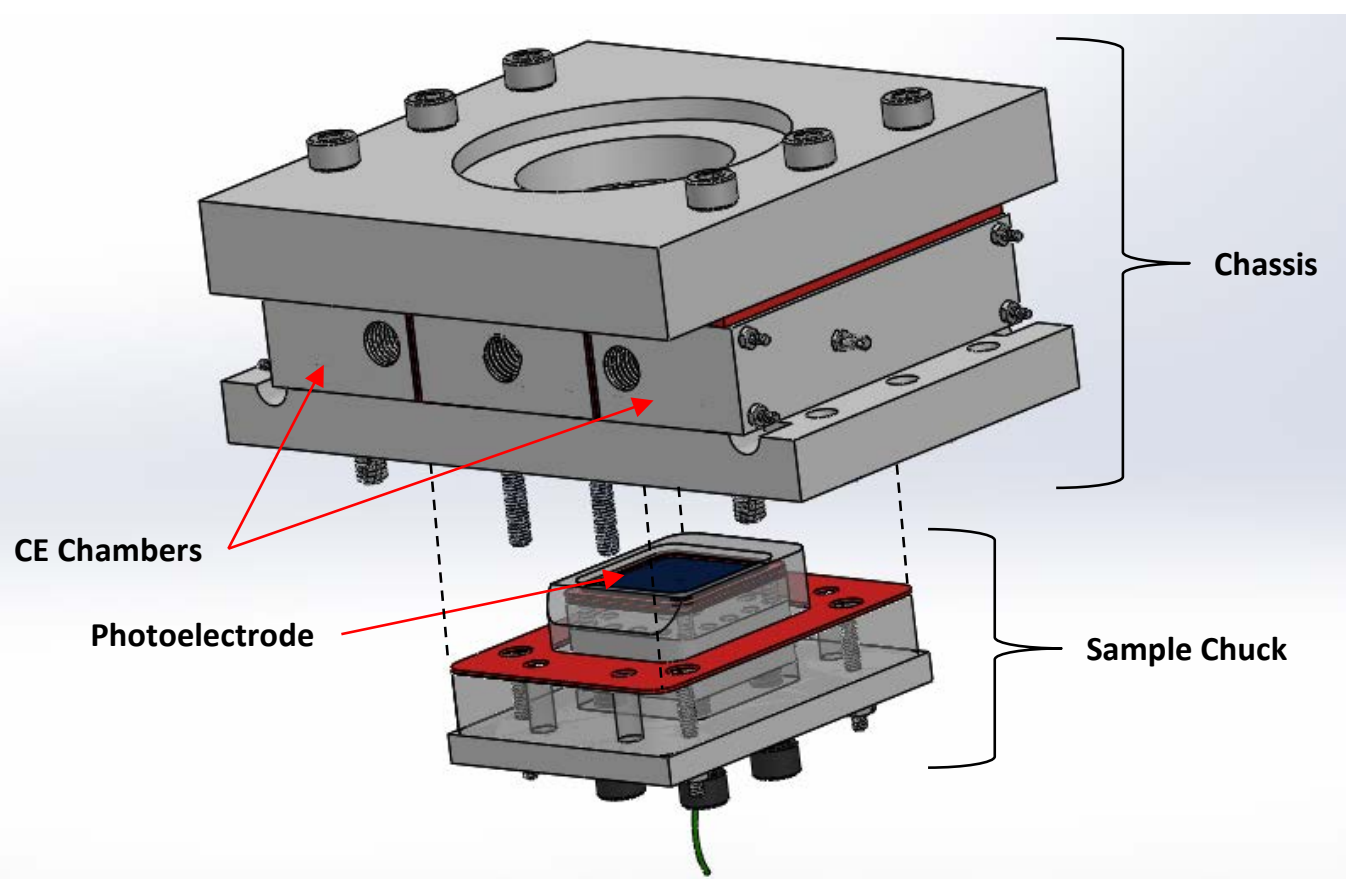

Figure 1: The assembled photoreactor with an exchangeable sample chuck removed from the bottom 


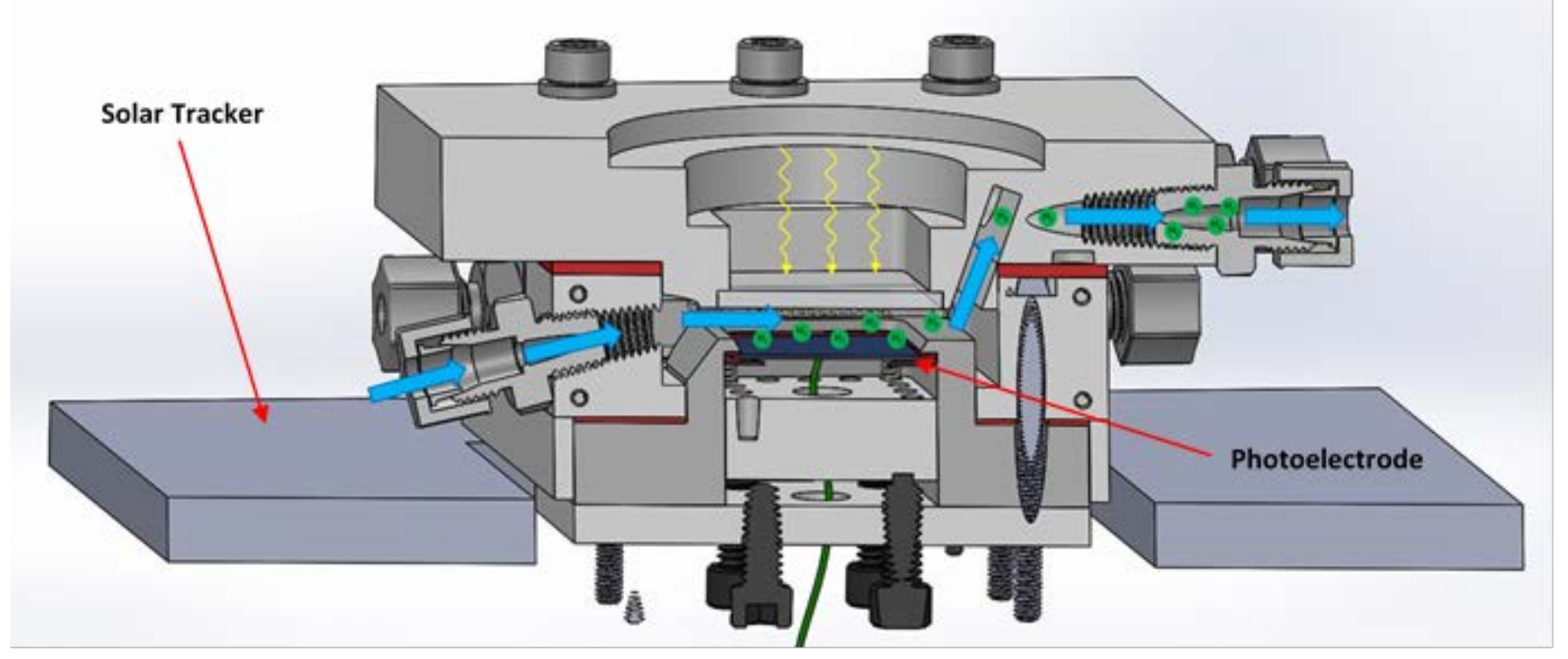

Figure 2: Photoreactor cross section showing the flow of electrolyte through the PE chamber (blue arrows), incident solar irradiance through the borosilicate window (yellow rays) incident on the photoelectrode (blue sheet), and generation of hydrogen gas from a photocathode (green circles). The dark anode is not visible in this cross-section. 


\section{List of Acronyms}

CE

EIS

HER

HFR

NREL

OER

PE
Counter electrode

Electrochemical impedance spectroscopy

Hydrogen evolution reaction

High frequency resistance

National Renewable Energy Laboratory

Oxygen evolution reaction

Photoelectrode 


\section{Photoreactor Assembly}

The following subsections describe the photoreactor components and their assembly.

\subsection{Assembling the CE Chambers}

1. Select a mesh-like CE that can accommodate a $1.5 \mathrm{~mm}$ hole or cut a hole if the CE is a solid sheet. It's important that the hole is close to the size of your titanium socket head screw to ensure good contact.

2. Place the titanium socket head screw (shown on the far right of Figure 3 ) through the hole in Step 1 and tighten it into the specialized screw. Check that the CE is in good electrical contact and mechanically secure.

3. Add a soft viton fluoroelastomer O-ring (McMaster, \#1284N103) to the opposite side of the specialized screw such that it is in contact with the screw's lip.

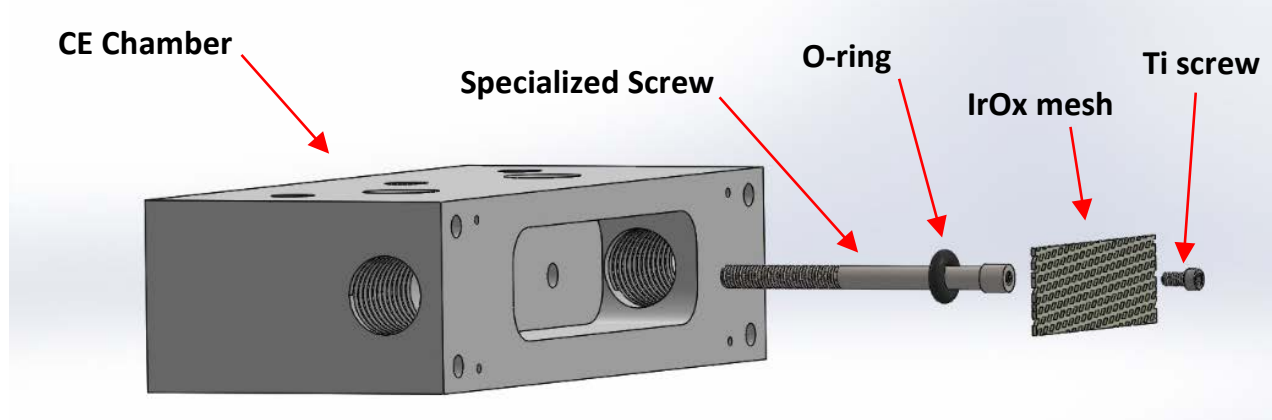

Figure 3. CE chamber expanded out with an iridium oxide mesh sample. The black o-ring is snuggly fit on the specialized screw.

4. With the CE and O-ring in place on the specialized screw, insert this assembly into the side of the CE chamber and add a washer and nut to the other side.

5. If using a fork terminal to make an electrical connection, place it between the washer and nut.

6. Tighten the nut and check that the O-ring has made a leak-tight seal.

7. After one CE chamber has been put together, assemble the second chamber (following the same instructions).

Caution: If the electrical contact between the CE and specialized screw is poor, the entire assembly will need to be taken apart to fix the connection. Check the series resistance of these metal components with a multimeter to ensure good connections before continuing assembly. Typical resistance values should be $<0.3 \mathrm{Ohm}$.

8. Add four alignment pins to each of the CE chambers (Figure 4). 
9. Cut four membrane gaskets and two Nafion ${ }^{\mathrm{TM}}$ membranes with the respective custommade dies.

10. In the following order, add a gasket, Nafion ${ }^{\mathrm{TM}}$ membrane, and gasket to the side of the $\mathrm{CE}$ chamber and push the alignment holes through their respective pins (Figure 4).

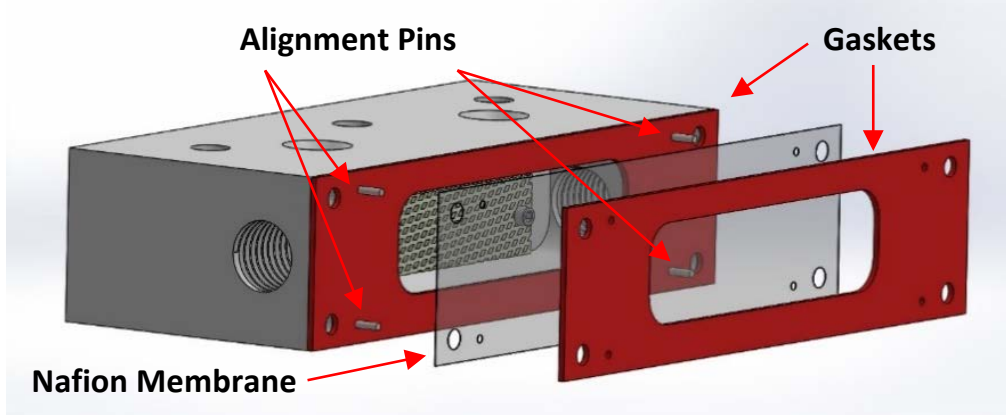

Figure 4. CE chamber with iridium oxide sample in place and gasket/Nafion membranes expanded out.

\subsection{Assembling the Photoreactor Body}

1. With the assembled CE chambers, connect them onto each side of the PE chamber and place four 135-mm long threaded titanium rods through the PE and CE chambers (Figure 5).

2. Add nuts to the titanium rods and lightly tighten them to keep the three chambers in one piece. Do not over tighten them as it will make it impossible to insert the top chamber and sample chuck into the PE chamber.

3. Insert four 2" hex head screws (McMaster part \#92314A205) into the top slots on the PE chamber (Figure 5)

4. If not done already, mount the borosilicate window in position on the top chamber. Secure and seal it to the top chamber by adding a very small amount of EopTek 302-3M epoxy to the border and sides. Allow the epoxy to fully cure before proceeding. 


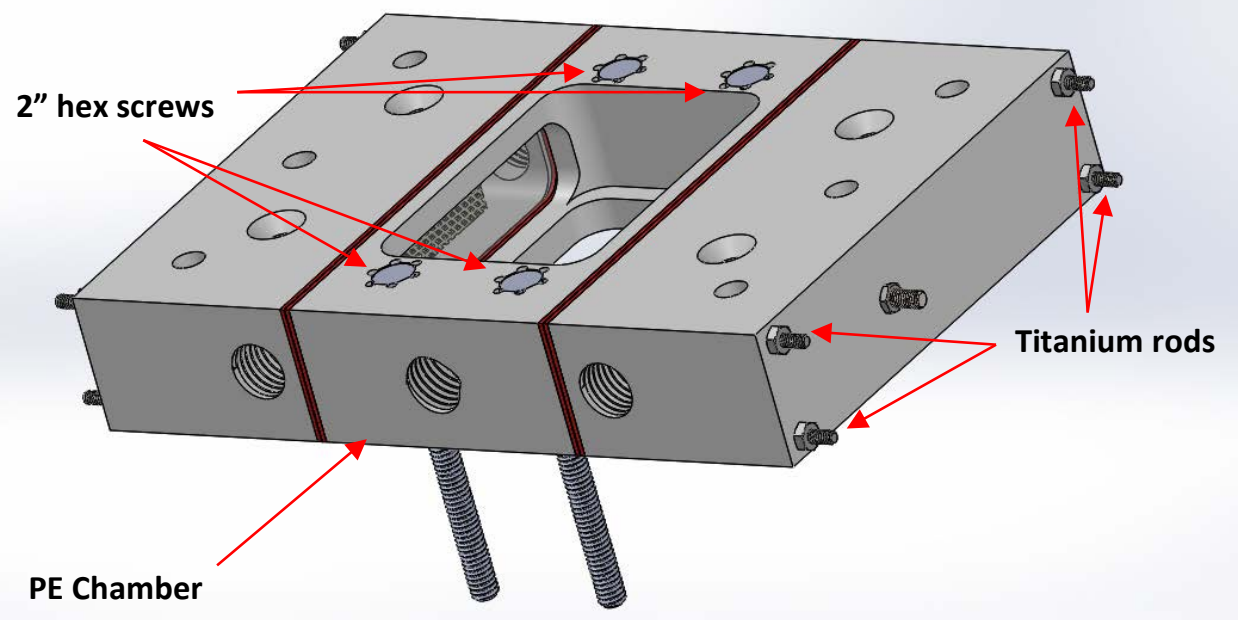

Figure 5. CE and PE chambers coupled together

5. Place a gasket on the bottom of the top chamber piece.

6. With the gasket and borosilicate window in place, insert the top chamber into the PE chamber.

7. Add six washers and socket head screws to the top of the top chamber and add the back plate (Figure 6, right) behind it. Lightly tighten the screws but wait until the sample chuck has been inserted to fully tighten them.
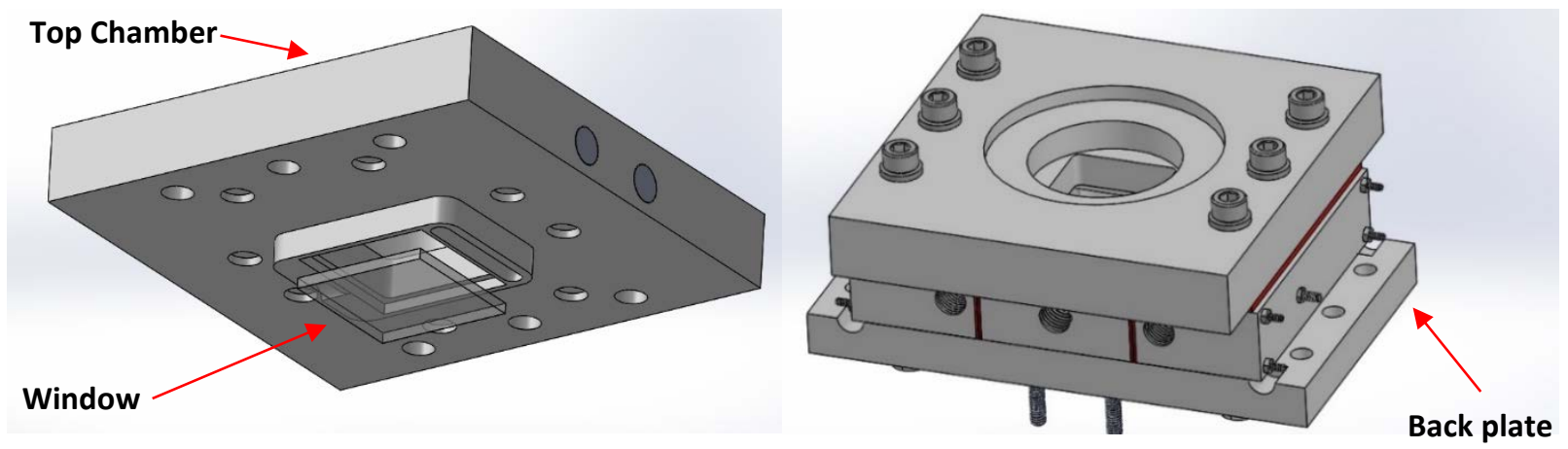

Figure 6. (Left) Bottom view of the top plate showing the window placement described in step 6. (Right) Top view of the assembled chassis.

\subsection{Assembling the Sample Chuck}

1. Cut three gaskets with the premade dies: two for the sample and one for mounting the sample chuck

2. Add one of the sample gaskets to the inside of the sample cavity

3. With a photoabsorber sample that has a conducive back contact, connect electrical wire to the back of it using silver paste or conductive epoxy. Once cured, mechanically strengthen the connection (e.g. with another epoxy such as Hysol 9462) to ensure a robust attachment.

4. Carefully place the sample against the gasket, checking that all sides of the sample are in contact with the gasket. 
5. Place a second gasket behind the sample, again checking that the sample is evenly overlapping on all sides.

6. Add the sample "balancer" (rigid piece of acrylic) behind the second gasket and place the contact wire through the middle of it. This piece will distribute the force from the springs to create a uniform sealing force.
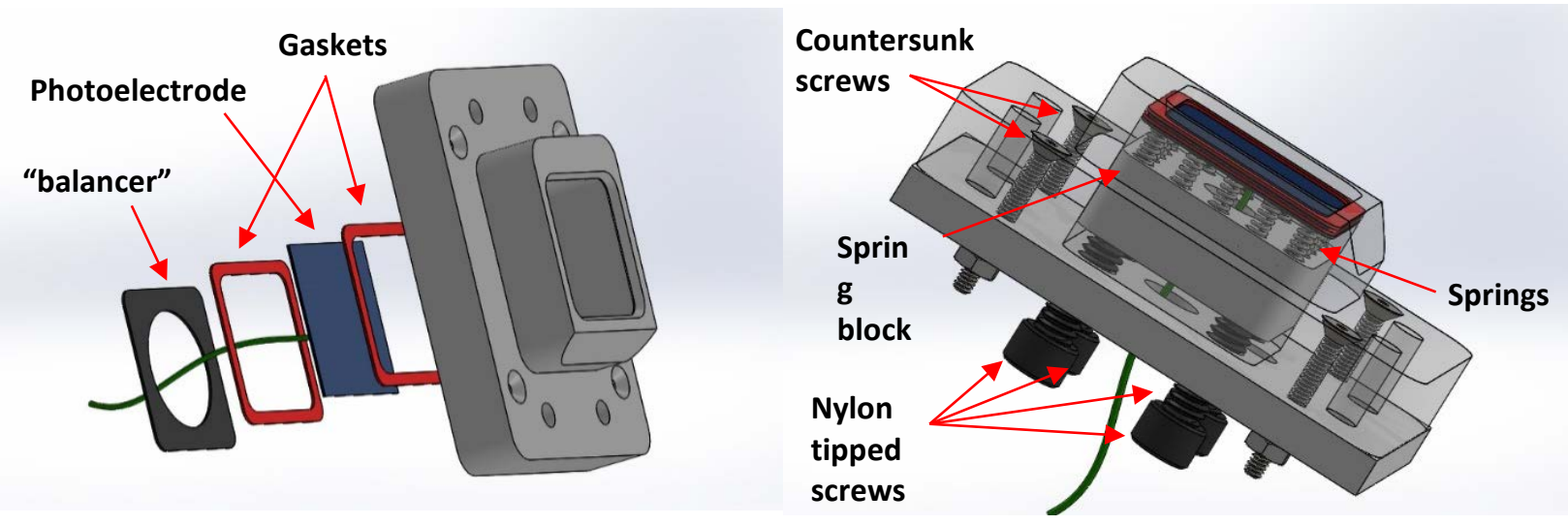

Figure 7: (Left) Top of the sample chuck with the $1^{\text {st }}$ gasket, photoelectrode sample, $2^{\text {nd }}$ gasket, and "balancer" shown from right to left. (Right) Final assembly of the sample chuck.

7. Place each spring in the holes around the perimeter of the spring block. If desired, a small amount of silicone can be used in each spring block hole to keep the springs secured in place. Allow the silicone to dry before moving on to the next step.

8. With the springs facing the sample, slide the spring block in and press the back plate up against it.

9. Add the four countersunk screws into the top of the sample chassis and tighten them with washers and nuts on the back plate.

10. Slowly start tightening the four nylon tipped screws into the back plate. Once the nylon tips engage the spring block, rotate them in small increments in a star pattern to maintain nearly uniform pressure on the sample. If one side is over tightened, it can cause the sample to break and/or a leak to form.

11. Place the gasket for the chuck atop of it (Figure 8). 

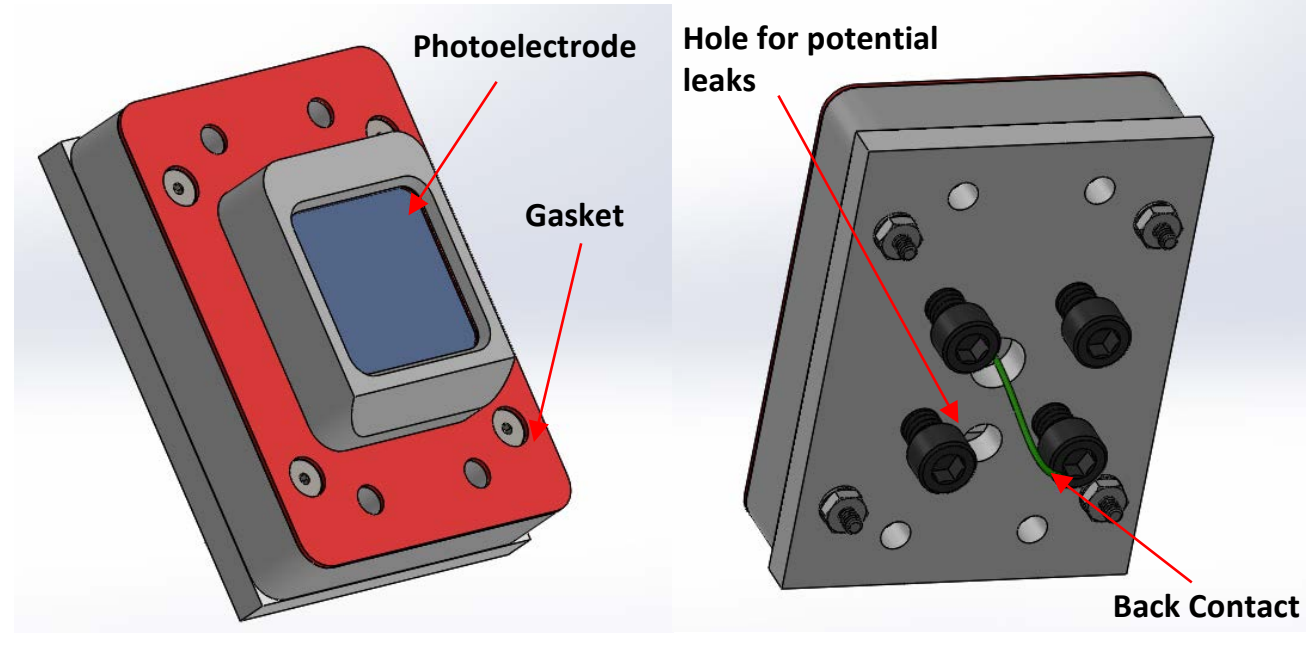

Figure 8: (Left) Sample chuck with top gasket (red) in place. (Right) Back of assembled sample chuck.

\subsection{Final Assembly}

1. Slide the sample chuck, with gasket, into the back of the preassembled photoreactor. The four holes in the assembled sample chuck will align with the 2" hex head screws from Figure 5.

2. Tighten the sample chuck into place with washers and nuts.

3. Finish tightening the nuts at each end of the $135 \mathrm{~mm}$ titanium rods on the sides of the $\mathrm{CE}$ chambers.

4. Wrap Teflon ${ }^{\mathrm{TM}}$ tape around tube fitting threads to create a leak-tight connection. The Teflon ${ }^{\mathrm{TM}}$ needs to be wrapped in the same direction as the threads.

5. Once the appropriate tubing lines have been attached to the tube fittings, the system is ready to be tested for leaks.

\section{Operating the Photoreactor}

\subsection{Measurement Preparation and Chuck Exchange}

1. Connect each tubing line to a reservoir, with the PE line circulating through one reservoir and the $\mathrm{CE}$ line through a second. Distinct reservoirs are required so that the hydrogen and oxygen produced remain separated.

2. The headspace of each reservoir should be vented to a laboratory exhaust or to a gas collection and quantification system with appropriate safety design and engineering controls.

3. Add deionized (DI) water to the reservoirs and pump the DI water through the photoreactor to check for leaks. The DI water flow rate and pressure should be greater than or equal to that of the electrolyte flow rate and pressure used later.

4. Run water through the system for 2-3 hours to ensure no leaks appear and that the reservoir levels remain balanced. In the absence of visible external leaks, a significant misbalance of reservoir levels indicates a leak in or around the membrane exists. 
5. Once it has been confirmed that the system is leak free, switch out the water for electrolyte and begin performing experiments.

6. If the chuck needs to be exchanged, drain the electrolyte, and then perform a rinse by running DI water through the system before any disassembly.
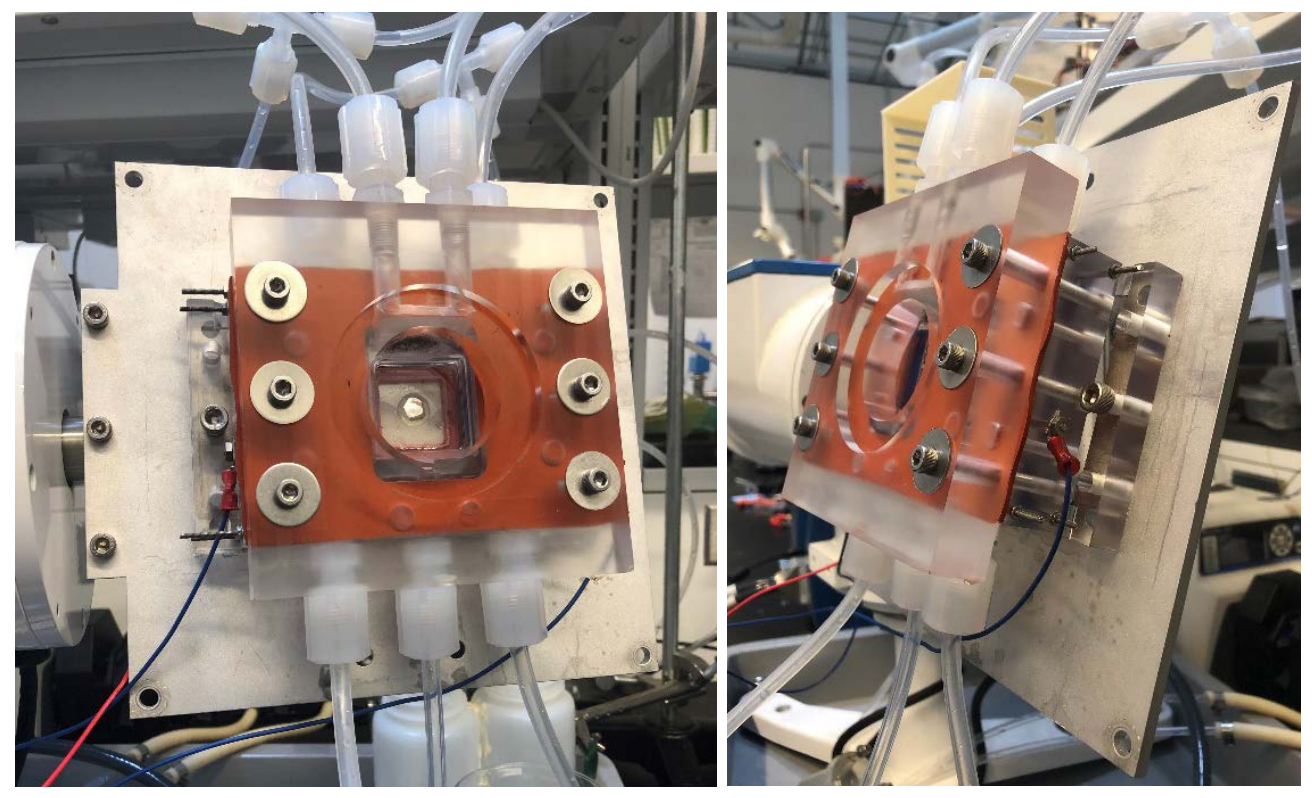

Figure 9: Front view (left) and side view (right) of the photoreactor mounted on a solar tracker. A piece of Pt foil is shown where the photoelectrode sits.

\section{Photoreactor Characterization Measurements}

\subsection{Experimental Setup}

The goal of these measurements was to quantify the total water splitting load and electrolyte resistance of two electrolytes commonly used in PEC measurements $(0.5 \mathrm{M}$ and $3 \mathrm{M}$ sulfuric acid). The photoreactor baseline performance was characterized by polarization curve and electrochemical impedance spectroscopy (EIS) measurements. A two-electrode measurement was performed with a BioLogic SP-300 potentiostat. The anode was an $\mathrm{IrO}_{2}$-coated Ti mesh; the cathode was a Pt foil. Measurements were taken at two different electrolyte flow rates of 20 $\mathrm{mL} / \mathrm{min}$ and $200 \mathrm{~mL} / \mathrm{min}$.

The high-frequency resistance (HFR) of an EIS measurement can be used as an approximation for the solution resistance ${ }^{2}$, here, because the resistance of the electrical connections in the photoreactor hardware was found to be negligible. The frequency range was set to $6 \mathrm{MHz}-0.5 \mathrm{kHz}$; lower frequencies are non-essential for the goal of this experiment. A DC current of $0.1 / 0.5 / 1 / 2 / 5 / 10 / 20 / 50 / 100 \mathrm{~mA}$ was applied, with a constant AC perturbation of 0.01 $\mathrm{mA}$.

\subsection{Method of HFR Determination}

For each EIS measurement, three points on either side of the Nyquist plot real axis (for a total of 6 points) were averaged to determine the intercept and HFR. Performance was characterized 
(Figure 10) using $0.5 \mathrm{M}$ and $3 \mathrm{M}$ sulfuric acid and at electrolyte flow rates of $20 \mathrm{~mL} / \mathrm{min}$ and 200 $\mathrm{mL} / \mathrm{min}$. The photoreactor baseline performance is better in $3 \mathrm{M}$ sulfuric acid, particularly for high current density, while the flow rate does not appear to significantly influence the baseline performance.

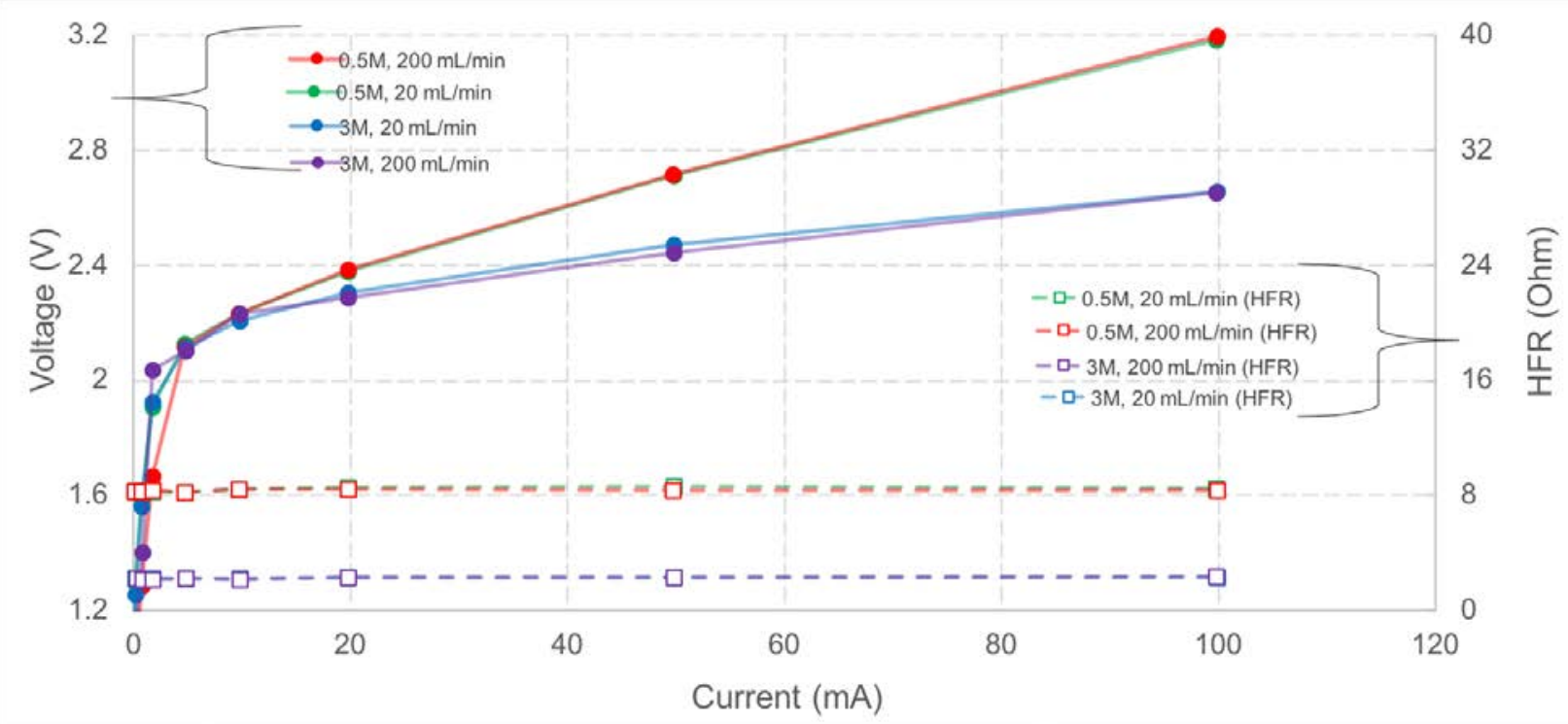

Figure 10: Polarization curves and EIS data detailing the current-voltage relationship and electrolyte resistance, respectively.

\section{Acknowledgements}

The authors gratefully acknowledge research support from the HydroGEN Advanced Water Splitting Materials Consortium, established as part of the Energy Materials Network under the U.S. Department of Energy, Office of Energy Efficiency and Renewable Energy, Hydrogen and Fuel Cell Technologies Office, under Award Number DE-EE-0008084. This work was authored by the National Renewable Energy Laboratory, operated by Alliance for Sustainable Energy, LLC, for the U.S. Department of Energy under Contract Number DE-AC36-08GO28308. The views expressed in the article do not necessarily represent the views of the DOE or the U.S. Government. The U.S. Government retains and the publisher, by accepting the article for publication, acknowledges that the U.S. Government retains a nonexclusive, paid-up, irrevocable, worldwide license to publish or reproduce the published form of this work, or allow others to do so, for U.S. Government purposes.

\section{References}

1. Deutsch, Todd G, et al. High-Efficiency Tandem Absorbers for Economical Solar Hydrogen Production. DOE Hydrogen and Fuel Cells Program, 2017.

2. "Electrochemical Impedance Spectroscopy." Electrochemical Methods and Applications, by Allen J. Bard and Larry R. Faulkner, Wiley-Interscience, 2000, p. 383. 


\section{Appendix A: Gasket dies}

\section{A.1 PE/CE Chamber Gasket:}

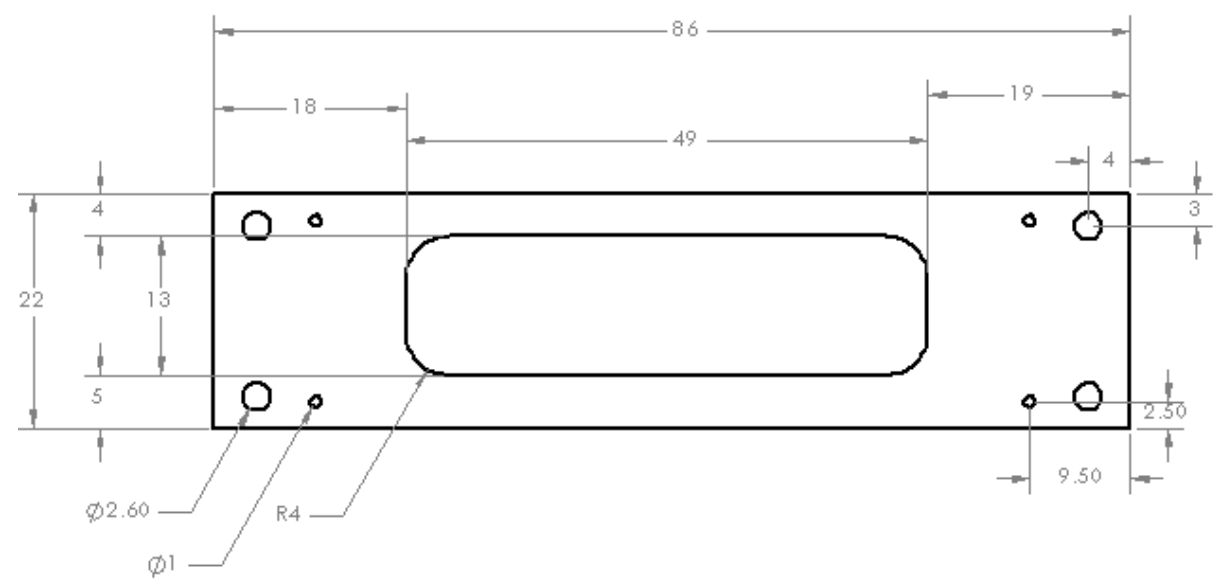

\section{A.2 Nafion $^{\mathrm{TM}}$ Membrane:}

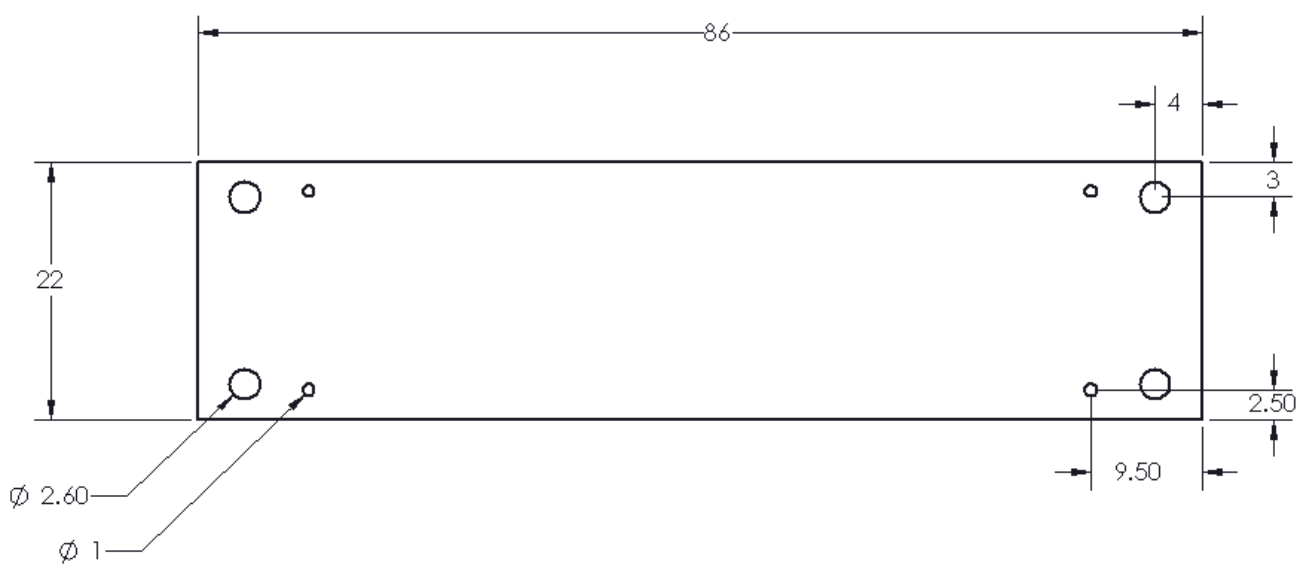




\section{A.3 Top Chassis:}

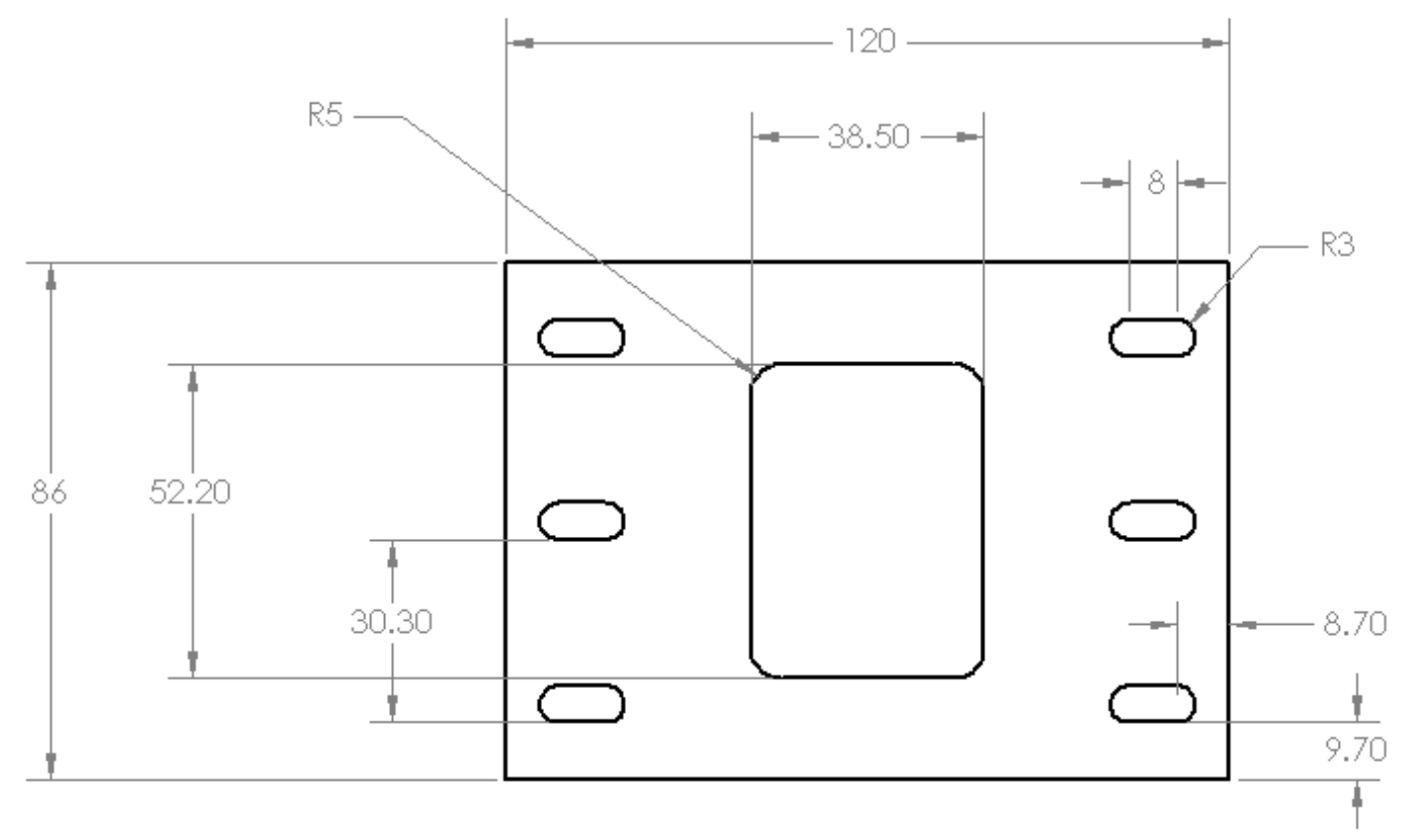

\section{A.4 Top Chuck Gasket:}

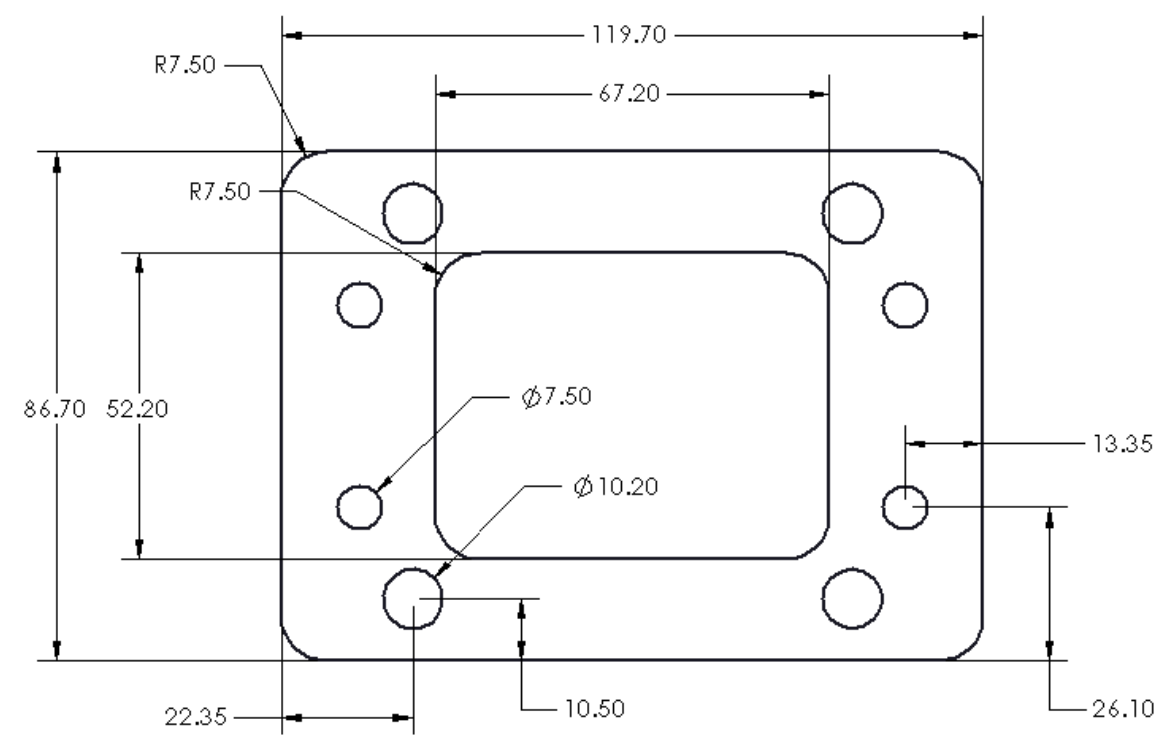




\section{A.5 Sample Gasket:}

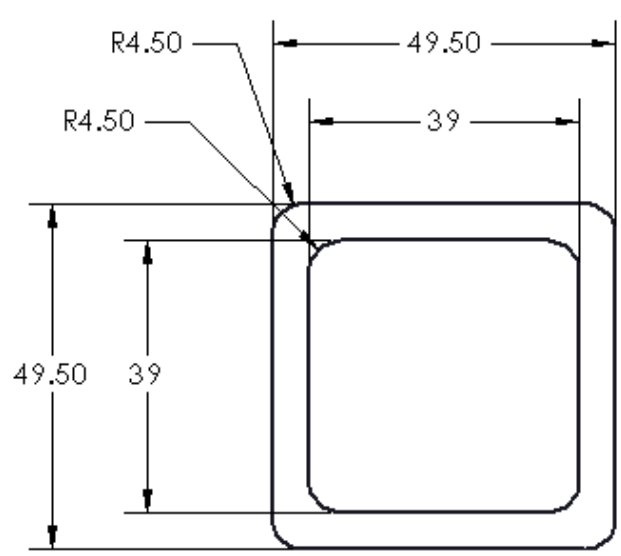




\section{Appendix B: General Design and Part Information}

- The photoreactor pieces are machined from polycarbonate, which is chemically resistant to acidic electrolytes.

- Five dies were machined; four to cut gaskets that are shown in red throughout this report, and one to cut the membranes

- Gaskets are cut from 1/32" or 1/64" thick sheets of fluorosilicone rubber (McMaster, \#2183T11)

- A Nafion ${ }^{\mathrm{TM}} 212$ membrane has been used in this set up to prevent the crossover of hydrogen and oxygen gas between chambers.

- Specialized screws were machined with external threads on one side and tapped holes on the other side, which is how the CEs are held in place. The screws were machined from grade 2 titanium.

- A 2" x 2" borosilicate window allows light to pass through the front of the photoreactor.

- To improve the flow of electrolyte, decrease the buildup of bubbles on the front window, and reduce the photon path length through electrolyte, three design characteristics have been implemented: the PE chamber inlet is tapped at an upwards angle of 30-degrees, the top part of the PE chamber is slanted downwards, and the sample chuck is slanted upwards (illustrated in Figure 8).

- In the top of the PE chamber, four hex head slots have been dog boned to keep the hex screws from spinning while loosening or tightening the chuck in place. This design allows the top chamber to remain attached when changing out the sample chuck.

- In case of a leak in the sample chuck, there is a small hole beneath the wire feed through that allows electrolyte to drain from the back cavity. This helps not only identify a leak sooner than later, but also prevents electrolyte from corroding the nylon-tipped screws and back contact wire. 